\title{
In Situ UV Measurements by MSL/REMS: Dust Deposition and Angular Response Corrections
}

\author{
A. Vicente-Retortillo ${ }^{1}$ G.M. Martínez ${ }^{1,2}$ • N.O. Rennó ${ }^{1}$. \\ M.T. Lemmon ${ }^{3}$ - M. de la Torre-Juárez ${ }^{4}$. \\ J. Gómez-Elvira ${ }^{5}$
}

Received: 9 November 2019 / Accepted: 9 July 2020 / Published online: 21 July 2020

(C) The Author(s) 2020

\begin{abstract}
Measurements by the REMS/UV sensor onboard the MSL Curiosity rover constitute the first in situ dataset of UV radiation flux at the surface of Mars. Due to its position on the Curiosity deck, the UV sensor has been directly exposed to dust deposition. Inaccuracies in the original angular response calibration functions have led to discrepancies between measured and physically-expected UV fluxes when the solar zenith angle $(\theta)$ relative to the rover frame is between $20^{\circ}$ and $55^{\circ}$. Here we present a methodology to correct UV fluxes when $\theta<55^{\circ}$ for both effects, and show results of the corrected data set for the first 2003 sols $\left(\sim 3\right.$ Martian Years, MY) of the MSL mission, from $\mathrm{L}_{\mathrm{s}} \sim 151^{\circ}$ in MY 31 to $\mathrm{L}_{\mathrm{s}} \sim 149^{\circ}$ in MY 34. Close to noon, when $\theta$ values are typically $<30^{\circ}$, relative differences between corrected and original UV fluxes are $\sim 35-40 \%$ on average. Outside hours close to noon, when $\theta$ is typically $>30^{\circ}$, relative differences are greater than $100 \%$. Measurements acquired when $20^{\circ}<\theta<55^{\circ}$ represent $\sim 45 \%$ of the whole dataset with $\theta<90^{\circ}$. UV fluxes generated in this study are available in the NASA Planetary Data System (https://atmos.nmsu.edu/PDS/data/mslrem_1001/DATA_UV_CORRECTED/), and are important to study the effect of UV radiation on the variability of atmospheric constituents, to recreate accurate UV doses for biological laboratory experiments, to perform combined analyses of satellite and ground-based measurements, and to allow comparisons of the UV radiation environment at different locations with the upcoming ExoMars 2020 and Mars 2020 missions.
\end{abstract}

Note by the Editor: This is a Special Communication, linked to the Topical Collection on the Mars Science Laboratory (MSL) published in Space Science Reviews (https://link.springer.com/journal/11214/170/1).

\footnotetext{
$\triangle$ A. Vicente-Retortillo alvarode@umich.edu

1 Department of Climate and Space Sciences and Engineering, University of Michigan, Ann Arbor, MI, USA

2 Lunar and Planetary Institute, Universities Space Research Association, Houston, TX, USA

3 Space Science Institute, College Station, TX, USA

4 Jet Propulsion Laboratory, California Institute of Technology, Pasadena, CA, USA

5 Centro de Astrobiología (INTA-CSIC), Madrid, Spain
} 
Keywords Mars · UV radiation · Dust deposition · Atmosphere

\section{Introduction}

The Rover Environmental Monitoring Station (REMS) onboard the Mars Science Laboratory (MSL) mission has a UV sensor (UVS) that has been measuring since 2012 the UV radiation flux at the surface of Mars for the first time in six bands between 200 and 380 $\mathrm{nm}$ (Gómez-Elvira et al. 2012). At the time of this writing, the UVS has completed more than 4.1 Martian years (MY) ( 2770 Martian days or sols) of measurements at Gale Crater $\left(4.6^{\circ} \mathrm{S}, 137.4^{\circ} \mathrm{E}\right)$, providing coverage ranging from diurnal to interannual time scales.

Because it is placed horizontally on the rover deck, the UVS is exposed directly to dust deposition (Smith et al. 2016; Vicente-Retortillo et al. 2018). This has led to significant time-dependent deviations in UV fluxes from nominal values. In addition, inaccuracies in the calibration function of the UVS angular response have led to discrepancies between measured and physically-expected UV fluxes when the solar zenith angle $(\theta)$ relative to the rover frame is between $20^{\circ}$ and $55^{\circ}$ (Vicente-Retortillo et al. 2017). In particular, the calibration function results in UV fluxes with a non-physical discontinuity at $\theta=30^{\circ}$.

Dust deposition and inaccuracies in the UVS angular response considerably complicate analyses of the highest-level (besides the dataset generated in this study) UVS data that are available in the NASA Planetary Data System (PDS), that is, the ENVRDR and MODRDR products. The ENVRDR products contain UV fluxes in units of $\mathrm{W} / \mathrm{m}^{2}$ for each UVS channel, while the MODRDR products contain identical data but with values of UV fluxes removed when $\theta$ is between $20^{\circ}$ and $55^{\circ}$ and when the rover or its arm move during measurements. To avoid uncertainties arising from these two effects, previous analyses of UVS measurements used lower-level UVS products (Smith et al. 2016; Vicente-Retortillo et al. 2017, 2018; Guzewich et al. 2019), from where UV fluxes recorded in the ENVRDR and MODRDR products are obtained. These lower-level products include ancillary data records containing the geometry of the rover and the Sun (ADR products) and output currents in units of nA measured by each photodiode (TELRDR products).

In this article we correct UV fluxes measured when $\theta<55^{\circ}$ for the effects of dust deposition and inaccuracies in the original angular response for the first 2003 sols of the MSL mission, generating corrected ENVRDR products and thus completing the MODRDR products by adding data when $20^{\circ}<\theta<55^{\circ}$. To perform this correction, we use ADR and TELRDR products, Mastcam opacities at $880 \mathrm{~nm}$ and UV fluxes simulated with the COMIMART radiative transfer model (Vicente-Retortillo et al. 2015).

UV fluxes generated in this study are important for studies of the effect of UV radiation on the variability of atmospheric gases such as methane and oxygen (Webster et al. 2018; Trainer et al. 2019), to improve our understanding of dust lifting on Mars and the dynamics processes related to it (Vicente-Retortillo et al. 2018; Newman et al. 2019), to further characterize dust particle size and optical properties (Lemmon et al. 2019; McConnochie et al. 2018), to recreate accurate UV doses for biological laboratory experiments, and to provide data accurate enough for comparisons of the UV radiation environment at different landing locations (Arruego et al. 2016; Rodriguez-Manfredi et al. 2014).

The organization of this article is as follows. In Sect. 2 we introduce the REMS/UVS onboard MSL. In Sect. 3 we explain the inaccuracies in UV fluxes caused by dust deposition and the use of the original calibration function of the angular response. In Sect. 4 we describe the methodology used to correct UV fluxes for both effects. In Sect. 5 we present the results of the correction and the associated uncertainties. In Sect. 6 we discuss the robustness of the correction. In Sect. 7 we present the summary and conclusions. 
Fig. 1 Spectral response of the six photodiodes (UVS channels) at $278 \mathrm{~K}$. The wavelength at maximum, $\lambda \max (\mathrm{nm})$, and the full width at half maximum, FWHM (nm), of the spectral response of the six channels are, respectively: 340 and 32 for UVA; 305 and 39 for UVB; 260 and 39 for UVC; 260 and 34 for UVD; 330 and 27 for UVE; and 290 and 98 for UVABC

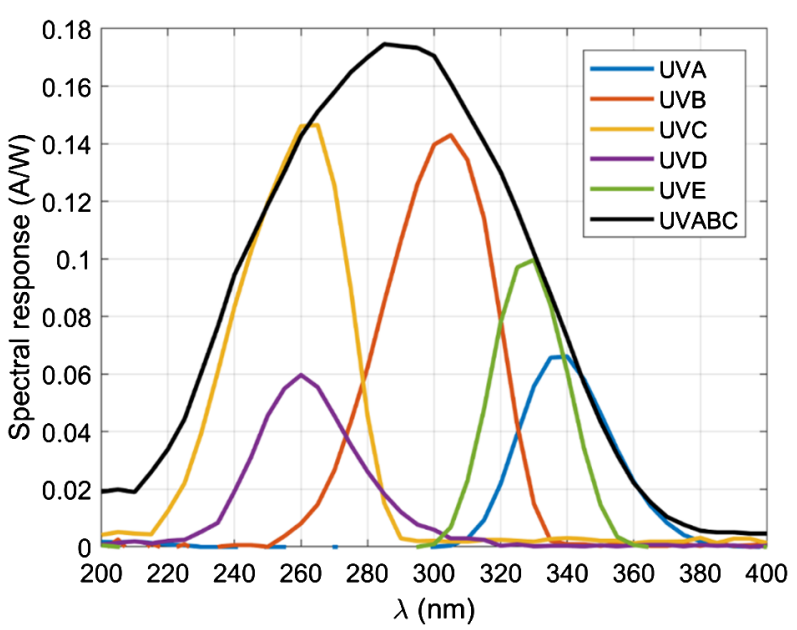

\section{The MSL/REMS UV Sensor}

The UVS consists of six photodiodes placed on the rover deck to measure UV flux in six different bands (Fig. 1). Channel ABC was designed to provide estimates of the total UV irradiance, A and B to compare the UV flux at the surface of Mars with that on Earth, C to provide a first order estimate of the level of biologically damaging irradiance, and D and $\mathrm{E}$ to match the two UV channels of the MARCI instrument onboard the Mars Reconnaissance Orbiter satellite (Gómez-Elvira et al. 2012).

The UVS nominal strategy for data acquisition consists of 5 minutes of measurements at $1 \mathrm{~Hz}$ every Mars hour, with at least one additional hour of $1 \mathrm{~Hz}$ measurement during each sol. Given additional available payload energy, the REMS measurements coverage can be extended by using the so-called extended blocks, which replace the nominal 5-minute blocks by blocks lasting one or more hours (Gómez-Elvira et al. 2014). This strategy has resulted in UVS measurements covering full diurnal cycles at $1 \mathrm{~Hz}$ every few weeks during more than 3 full annual cycles.

Besides the new corrected dataset generated in this study (hereinafter called UVRDR products), the highest-level REMS UVS data available in the NASA PDS are the ENVRDR and MODRDR products, followed by the ADR and TELDRD products. These data sets contain, among others, the following quantities:

1. ENVRDR products: UV fluxes in units of $\mathrm{W} / \mathrm{m}^{2}$ for each UVS channel.

2. MODRDR products: Similar to the ENVRDR products, but with values of UV fluxes removed when the solar zenith angle relative to the rover frame is between $20^{\circ}$ and $55^{\circ}$ and when the rover or its arm are moving.

3. ADR products: Rover location, pitch, yaw and roll; Sun position relative to the rover frame; azimuth and elevation angles of the masthead.

4. TELRDR products: Photodiode output currents in units of $n A$ measured by each UVS channel.

In this article, we refer to the zenith and azimuth angles relative to the rover frame. As described in the label files of the REMS directory in the NASA PDS, the origin of this frame is on the rover deck, between the middle wheels of the rover; the positive $\mathrm{X}$-axis points to the front of the rover and the positive $\mathrm{Z}$ axis points up. The solar zenith angle is the angle 
Fig. 2 (Top) MAHLI images of the REMS/UVS on sols 36 $\left(\mathrm{L}_{\mathrm{S}}=170^{\circ}\right.$ in MY 31) (left) and $808\left(\mathrm{~L}_{\mathrm{s}}=233^{\circ}\right.$ in MY 32) (right). Dust patterns are caused by circular magnetic rings. (Bottom) Diurnal evolution of UVB photocurrents on sols 76 (blue) and 745 (red), separated by one Martian year $\left(\mathrm{L}_{\mathrm{s}} \sim 193.5^{\circ}\right)$ and with roughly the same atmospheric opacity $(\tau \sim 0.75)$
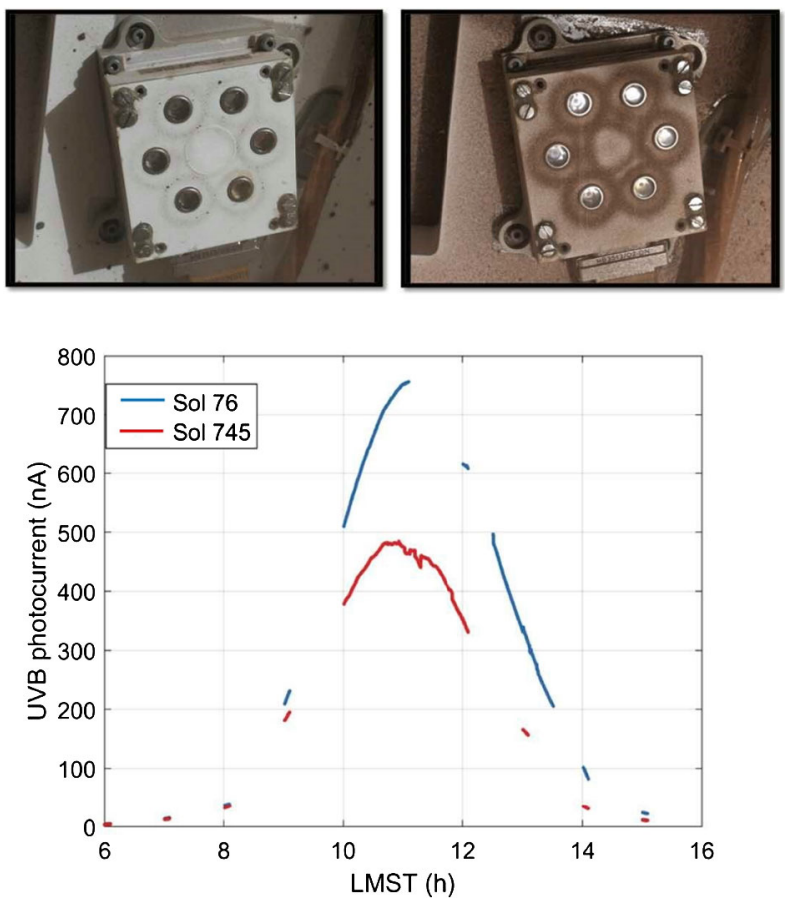

between the direction of the Sun and the positive Z-axis; its value is 0 when the Sun is at the zenith of the frame. The solar azimuth angle is the angle between the positive $\mathrm{X}$-axis and the orthogonal projection of the Sun onto the XY plane, and it increases in the counterclockwise sense about the positive Z-axis. Masthead elevation angles of $1^{\circ}$ and $91^{\circ}$ represent the rover looking down and forward, respectively. Masthead azimuth angles of $-179^{\circ},-89^{\circ}$ and $1^{\circ}$ represent rover looking forward, to the right, and backward, respectively.

The UV fluxes stored in the ENVRDR and MODRDR products are obtained using geometric information (ADR products), output currents measured by each photodiode (TELRDR products), and the original angular response calibration functions applied to such output currents. In Sect. 3 we show that TELRDR products are strongly affected by dust deposition and that the original angular responses present a non-physical discontinuity at $\theta=30^{\circ}$, both effects resulting in significant inaccuracies in the derived UV fluxes in the ENVRDR and MODRDR products.

\section{Inaccuracies in MSL/REMS UV Fluxes}

\subsection{Dust Deposition}

Due to its horizontal placement on the rover deck, the UVS has been directly exposed to dust deposition. This dust deposition is illustrated in Fig. 2, which shows images of the UVS at the beginning of the mission on sol 36 (top left) and more than one Martian year later on sol 808 (top right). Dust causes a reduction in measured UV fluxes. As an example, Fig. 2 (bottom) shows UVB photocurrents measured on sols 76 (blue curve) and 745 (red curve). Since Mastcam dust opacity values and the Sun-Mars distance were roughly the same on 

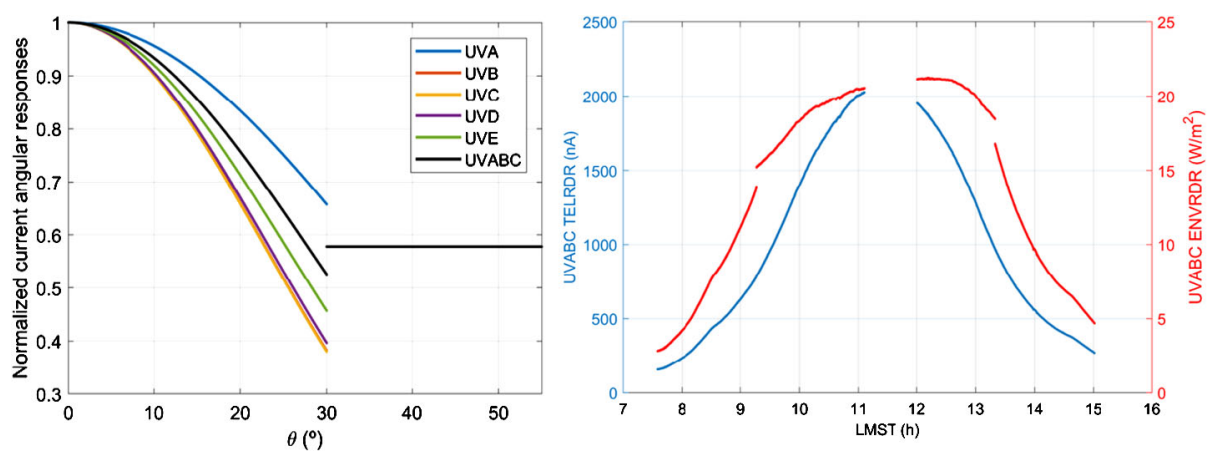

Fig. 3 (Left) Normalized angular response calibration functions used to generate ENVRDR and MODRDR products currently available in NASA's Planetary Data System as a function of the solar zenith angle. The six calibration functions show a discontinuity at $\theta=30^{\circ}$ and a constant value (which is the same for the six channels) beyond this angle. The nominal field of view of the sensor is $\pm 30^{\circ}$ (Gómez-Elvira et al. 2012), and the calibration was performed within that range. However, incident radiation with zenith angles $>30^{\circ}$ can reach the photodiode after internal reflections. This effect is considered in the new angular responses (Fig. 6). (Right) The UVABC photocurrents measured on sol 91 (blue, TELRDR data) vary smoothly with solar zenith angle, but the processed UVABC fluxes (red, ENVRDR data) contain a discontinuity when $\theta=30^{\circ}$. This discontinuity is caused by the discontinuity in the instrument angular response calibration function shown on the left (black)

both sols $\left(\tau=0.75\right.$ and $\left.\mathrm{L}_{\mathrm{s}} \sim 193.5^{\circ}\right)$, similar UVB levels are expected. However, UVB levels measured during the second Martian year of the mission are significantly lower due to dust deposited on the UVS. A similar behavior is found in other UV channels.

Design constraints ruled out any active protection system for the UVS (Gómez-Elvira et al. 2014). To mitigate dust deposition, each photodiode was embedded in a samarium cobalt magnetic ring to deflect the trajectories of the magnetic portion of falling dust, with an additional ring located at the center of the six photodiodes (Fig. 2, top right). This is similar to the sweep magnet of the Magnetic Properties Experiment on the Mars Exploration Rover (MER) (Madsen et al. 2003). Regardless, degradation of the UVS due to dust deposition has led to significant deviations from nominal values.

\subsection{Angular Response Functions}

Inaccuracies in the original angular response calibration function of each UVS channel lead to physically-inconsistent variations in UV fluxes (ENVRDR products) when the solar zenith angle relative to the rover frame is between $20^{\circ}$ and $55^{\circ}$. In particular, UV fluxes show a non-physical discontinuity at $\theta=30^{\circ}$ caused by the use of two different calibration functions that do not converge to the same value at $30^{\circ}$. Additionally, differences between the original and here-obtained calibration functions when $20^{\circ}<\theta<30^{\circ}$ can cause significant departures in the derived UV fluxes (more details in Sect. 4.2).

Figure 3 (left) shows the normalized angular response calibration function of each UVS channel as a function of the solar zenith angle. These functions are used to convert photodiode currents (TELRDR products) into UV fluxes (ENVRDR products). As an example, the UVABC fluxes obtained when this angular response calibration function is applied to the output currents measured on sol 91 are shown in Fig. 3 (right). The discontinuity in the UVABC fluxes (red curve) at $\theta=30^{\circ}$ is caused by the discontinuity in the angular response calibration function. However, values of the photodiode output current (blue curve) show a consistent behavior when $\theta=30^{\circ}$. 


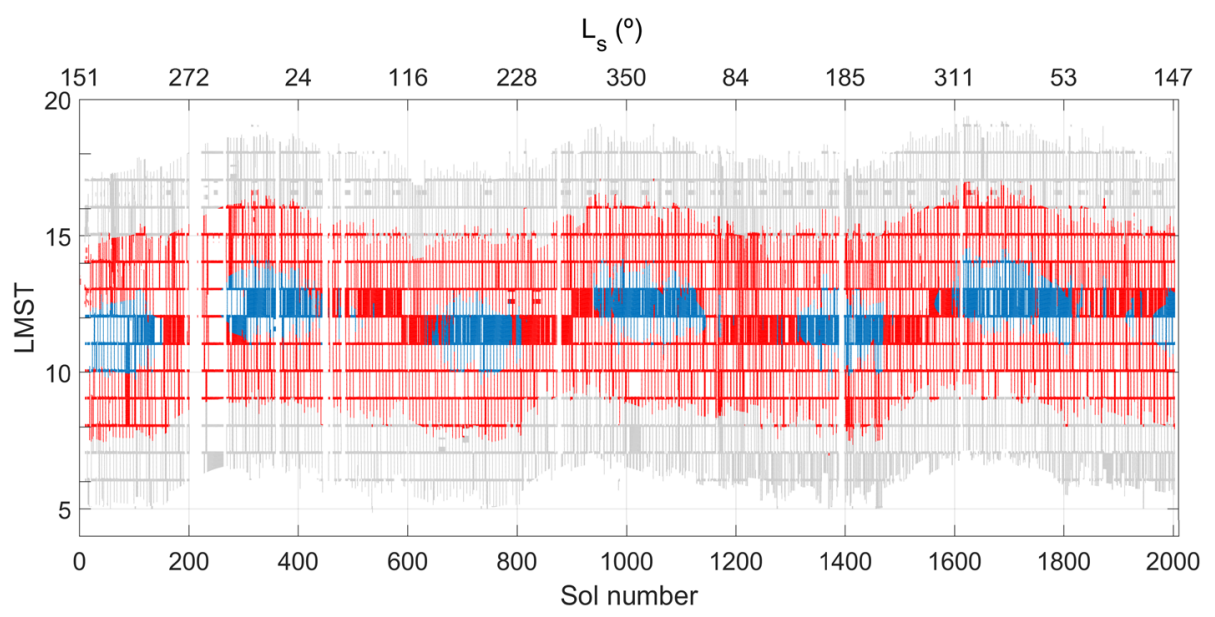

Fig. 4 Temporal coverage of the REMS UVS instrument as a function of sol number and local mean solar time (LMST). In blue, measurements when $\theta<20^{\circ}$, which are included in both ENVRDR and MODRDR products. In red, measurements when $20^{\circ}<\theta<55^{\circ}$, which have been removed from the most processed MODRDR data products. In gray, measurements when $\theta>55^{\circ}$, which are available in both ENVRDR and MODRDR products

Measurements acquired when $20^{\circ}<\theta<55^{\circ}$ (i.e., UV fluxes removed in the MODRDR products) represent $\sim 45 \%$ of the whole set of UVS data with $\theta<90^{\circ}$. This is shown in Fig. 4, where the temporal coverage of the UVS is represented as a function of the sol number and Local Mean Solar Time (LMST). UV measurements taken when $20^{\circ}<\theta<$ $55^{\circ}$ are colored in red, while those taken when $\theta<20^{\circ}$ are colored in blue. Finally, UV measurements taken at $\theta>55^{\circ}$ are colored in gray. Throughout the mission and in particular around the solstices $\left(\mathrm{L}_{\mathrm{s}}\right.$ around $90^{\circ}$ and $\left.270^{\circ}\right)$, measurements acquired when $20^{\circ}<\theta<55^{\circ}$ include a significant fraction of the diurnal cycle.

Here we correct UV fluxes taken when $\theta<55^{\circ}$ (blue and red time coverage in Fig. 4) for inaccuracies in the angular response. Details of this correction are given in Sect. 4.2.

\section{Methodology}

In this section, we describe the methodology used to generate corrected UV fluxes. First, we describe the calculation of a function that accounts for the effects of dust deposition. Second, we describe the methodology to obtain new angular responses for each channel. Finally, we describe the conversion from electrical units to UV fluxes.

\subsection{Dust Deposition Correction}

We quantify the effect of dust deposited on the UVS by calculating a dust correction factor (DCF). This quantity is defined as the fraction of the incoming UV radiation at the surface that reaches the photodiode through dust accumulated on the sensor, with respect to the fraction at the beginning of the mission. The DCF has a value of 1 at the beginning of the mission (when the sensor was clean) and, for example, a value of 0.7 indicates that only $70 \%$ of the UV flux at the surface is transmitted through the dust accumulated on the window of the sensor. To calculate the DCF, we use TELDRDR and ADR products (Sect. 2). 
In addition, we use Mastcam opacities and UV fluxes simulated with the numerical model COMIMART (Vicente-Retortillo et al. 2015).

We describe next the steps followed to calculate the DCF. These steps were presented in Vicente-Retortillo et al. (2018), where the DCF for each UVS channel was obtained for the first 1648 sols of the MSL mission. Here, we provide greater detail and extend the calculation to the first 2003 sols of the mission.

Since the correction presented here relies on the comparison of measurements performed for certain conditions on different sols, we first discard measurements in the TELRDR products acquired when the masthead of the rover was not in its most typical position (elevation $\sim 43^{\circ}$ and azimuth $\sim-179^{\circ}$ relative to the rover frame). This condition is very important, as the position of the masthead can affect significantly the amount of radiation that reaches the photodiode. Second, we discard measurements acquired for solar azimuth angles between $-100^{\circ}$ and $10^{\circ}$ (the quadrant in which the mast is, with an additional margin) to avoid the effect of the shadows cast by the masthead and mast of the rover (Vicente-Retortillo et al. 2017). Third, we discard measurements during drives and when the solar zenith angle was above $45^{\circ}$ to minimize uncertainties in the retrievals. Finally, the remaining measurements are corrected accounting for temperature variations in the spectral response of the photodiodes (Gómez-Elvira et al. 2012) and stored in a tridimensional table as a function of sol number and solar zenith and azimuth angles relative to the rover frame (hereinafter we will refer to the relative solar position simply as solar position).

Then, we calculate relative variations in the amount of accumulated dust between pair of sols with available Mastcam opacity measurements. Since the reflectance (and transmittance) of surfaces with deposited dust are expected to depend not only on the amount of dust but also on the solar position among other factors (Johnson et al. 2015), we search for measurements performed under the same solar zenith and azimuth angles for each pair of selected sols. If there are not matches between the two sols, their relative DCF is not computed. If there are matches, we select the solar position that both sols have in common with the highest measured values of output currents. To make measurements comparable, we apply factors that account for the Sun-Mars distance and for the opacity on both sols. We perform this normalization using UV fluxes simulated as a function of opacity and solar zenith angle using the COMIMART radiative transfer model; this model uses aerosol radiative properties retrieved from MARCI observations (Wolff et al. 2010). We obtain the relative DCF (RDCF) of the second sol (in chronological order) with respect to the first one (considered as a reference) by dividing the corresponding normalized measurements as:

$$
R D C F_{j, i}=\frac{F_{j} / C_{j}\left(\tau_{j}, L_{s, j}, \theta_{j, i}, \lambda\right)}{F_{i} / C_{i}\left(\tau_{i}, L_{s, i}, \theta_{j, i}, \lambda\right)},
$$

where $F$ is the measured UV flux, $C$ is a factor that accounts for the Sun-Mars distance (which depends on the solar longitude, $L_{s}$ ) and the effect of the suspended dust (which depends on opacity, $\tau$, and solar zenith angle, $\theta$, and wavelength of each UV channel, $\lambda$ ), i indicates the reference sol (from 1 to $\mathrm{N}$, where $\mathrm{N}$ is the total number of sols with Mastcam opacities) and $\mathrm{j}$ indicates the sol for which the RDCF is calculated (from $\mathrm{i}$ to $\mathrm{N}$ ).

Our next step is to normalize the obtained temporal series of RDCF. We first select a sol, hereinafter sol s. Second, we create a data subset (subset 1) with all the RDCFs that were obtained using reference sols for which a RDCF for sol s was obtained. Third, we create another subset (subset 2) with all the RDCFs that were obtained using reference sols for which a RDCF was obtained using sol $s$ as a reference. These two subsets $(\mathrm{S})$ for the reference sol s are:

$$
S_{s, 1}=\left\{R D C F_{s, l} \mid 1 \leq l<s\right\}
$$




$$
S_{s, 2}=\left\{R D C F_{m, s} \mid s \leq m \leq N\right\}
$$

Fourth, we normalize the RDCFs of both subsets with respect to the RDCF on sol s, obtaining a number of temporal series of normalized RDCF (NRDCF):

$$
\begin{gathered}
N R D C F_{s, k, i}=\frac{R D C F_{i, l}}{R D C F_{s, l}} \quad 1 \leq k \leq N_{l} \\
N R D C F_{s, k+N_{l}, i}=R D C F_{i, m} \cdot R D C F_{m, s} \quad 1 \leq k \leq N_{m}
\end{gathered}
$$

Here, $N_{l}$ and $N_{m}$ are the number of elements in $S_{s, 1}$ and $S_{s, 2}$, respectively. Finally, for each sol of these temporal series, we select the DCF obtained with the lowest value of the maximum solar zenith angle involved in the calculation. As an example, let us consider three sols (sol 1 being the reference), and that for sol 3 we have two relative DCFs. The first one $\left(\mathrm{RDCF}_{3,1}\right)$ is obtained by comparing measurements on sols 1 and 3 . The second one $\left(R D C F_{3,1}^{*}\right)$ is obtained by comparing measurements on sols 2 and 3, but a comparison between sols 1 and 2 is also made in order to obtain a value for sol 3 referenced to sol 1 (therefore, there are two solar zenith angles involved in this second calculation, $\theta_{3,2}$ and $\left.\theta_{2,1}\right)$ :

$$
\begin{gathered}
R D C F_{3,1}=\frac{F_{3} / C_{3}\left(\tau_{3}, L_{s, 3}, \theta_{3,1}, \lambda\right)}{F_{1} / C_{1}\left(\tau_{1}, L_{s, 1}, \theta_{3,1}, \lambda\right)} \\
R D C F_{3,1}^{*}=\frac{R D C F_{3,2}}{R D C F_{1,2}}=\frac{R D C F_{3,2}}{1 / R D C F_{2,1}}=\frac{\frac{F_{3} / C_{3}\left(\tau_{3}, L_{s, 3}, \theta_{3,2}, \lambda\right)}{F_{2} / C_{2}\left(\tau_{2}, L_{s, 2}, \theta_{3,2}, \lambda\right)}}{\frac{F_{1} / C_{1}\left(\tau_{1}, L_{s, 1}, \theta_{2,1}, \lambda\right)}{F_{2} / C_{2}\left(\tau_{2}, L_{s, 2}, \theta_{2,1}, \lambda\right)}}
\end{gathered}
$$

If the solar zenith angle for the comparison between sols 1 and 3 is smaller than the largest value of the two zenith angles involved in the second calculation, that is, if $\theta_{3,1}<\max$ $\left\{\theta_{3,2} ; \theta_{2,1}\right\}$, then we use $\mathrm{DCF}_{3,1}$; otherwise, we would use $D C F_{3,1}^{*}$. After performing these selections, we obtain a final temporal series of normalized relative dust correction factors using sol $\mathrm{s}$ as a reference $\left(\mathrm{NRDCF}_{\mathrm{s}}\right)$.

We repeat the process described in the last four paragraphs for each sol with Mastcam opacity retrievals, obtaining a temporal series relative to each sol with opacity measurements. Then, the DCF for a given sol $\mathrm{s}$ is calculated as follows:

$$
D C F_{s}=\overline{\Gamma_{s, i}} \quad 1 \leq i \leq N,
$$

where:

$$
\begin{gathered}
\Gamma_{s, r}=\mathrm{B}_{s, r} / \mathrm{B}_{s, 1} \quad 1 \leq r \leq N \\
\mathrm{~B}_{s, r}=\overline{\beta_{s, j}} \quad 1 \leq j \leq N \\
\beta_{j, s} \equiv \beta_{s}=N R D C F_{s} \cdot \overline{\left(\frac{N R D C F_{r}}{N R D C F_{s}}\right)}
\end{gathered}
$$

In order to improve the coverage and quality of the correction, we smooth DCF values by applying a weighting function with a triangular shape to the values within 50 sols from each sol. Finally, we normalize the smoothed version to the first sol for which Mastcam opacity measurements are available, and set the DCF to 1 for the previous sols. 
Fig. 5 Dust correction factor as a function of solar longitude for the UVABC channel in MY 31 (blue), 32 (black), 33 (red) and 34 (green). The remaining UV channels behave similarly (see Sect. 6)

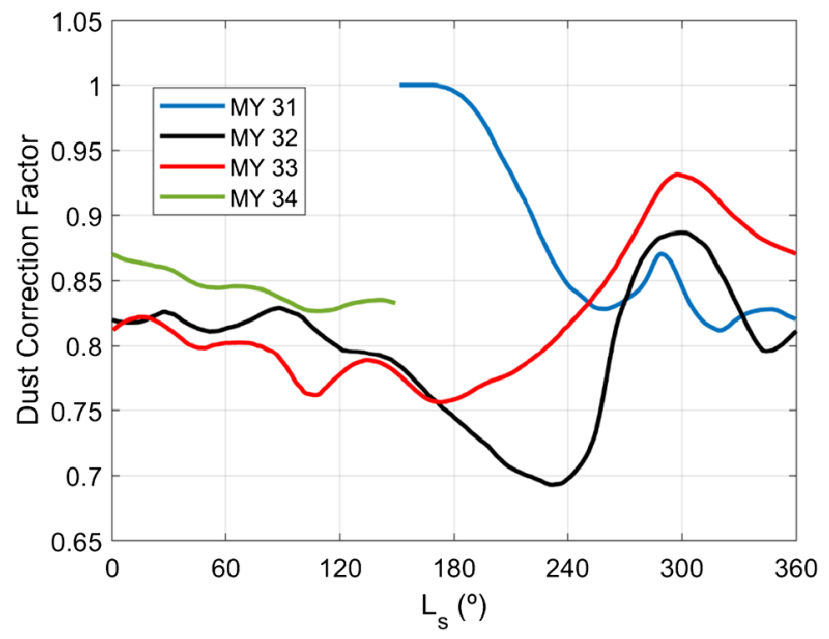

Figure 5 shows values of the DCF for the UVABC channel as a function of $\mathrm{L}_{\mathrm{s}}$ during the first 2003 sols of the MSL mission. The DCF for each of the other channels behaves similarly (Sect. 6). The DCF follows a seasonal cycle, with net dust removal (increasing DCF) during the perihelion season until $\mathrm{L}_{\mathrm{s}} \sim 300^{\circ}$, followed by net dust accumulation (decreasing DCF) until the end of the aphelion season $\mathrm{L}_{\mathrm{s}} \sim 300^{\circ}-180^{\circ}$. A discussion on the atmospheric mechanisms driving the variability of the DCF is given in Vicente-Retortillo et al. (2018).

Results are very similar to those presented in Vicente-Retortillo et al. (2018), with differences between $-2 \%$ and $+3 \%$. The differences arise from a slight change in the methodology to improve the accuracy of the DCF: the factors to compensate the effects of atmospheric opacity are now computed using the radiative properties at the corresponding wavelengths for each channel according to Fig. 1, instead of using the same factor for the six channels.

Figure 5 highlights the importance of correcting for dust deposition. The DCF shows values below 0.9 (indicating that the UV fluxes are underestimated by more than a $10 \%$ ) shortly after sol $100\left(\mathrm{~L}_{\mathrm{s}} \sim 208^{\circ}\right)$ in MY 31 (blue curve). After the abrupt decrease of the DCF during MY 31, the effect of accumulated dust exacerbated during MY 32, reaching a maximum attenuation of $30 \%(\mathrm{DCF}=0.7)$ at $\mathrm{L}_{\mathrm{s}} \sim 240^{\circ}$ (black curve). Following a net dust removal period, attenuation decreased to values slightly above $10 \%$ at $\mathrm{L}_{\mathrm{s}} \sim 300^{\circ}$. A similar pattern is observed in MY 33 (red curve), with a minimum in dust accumulation also observed at $\mathrm{L}_{\mathrm{s}} \sim 300^{\circ}$ and a maximum at $\mathrm{L}_{\mathrm{s}} \sim 180^{\circ}$.

Interestingly, our results indicate that during the perihelion season the UVS was cleaner in MY 33 than in MY 32. Mastcam opacities were similar in both perihelion seasons (Lemmon et al. 2019), with a maximum of $\sim 1.5$ at $\mathrm{L}_{\mathrm{s}} \sim 230^{\circ}$, a relative minimum of $\sim 0.8$ at $\mathrm{L}_{\mathrm{s}} \sim 300^{\circ}$, and a secondary maximum of $\sim 1.2$ at $\mathrm{L}_{\mathrm{s}} \sim 330^{\circ}$; this suggests that the interannual difference in the amount of accumulated dust is likely caused by a change in the frequency and strength of the dust lifting and removal mechanisms: daytime convective vortices and nighttime winds (Vicente-Retortillo et al. 2018). Analyses of REMS pressure perturbations and mesoscale modeling results show that this interannual variability is caused by the spatial variations in the conditions that lead to dust lifting (Vicente-Retortillo et al. 2018; Newman et al. 2019). The rover moved from the crater trench during the aphelion season of MY 32 to the slopes of Aeolis Mons during that of MY 33, resulting in an increase of the difference between surface and air temperatures and in the strength of upslope winds. 
Both effects, in turn, cause an increase in the sensible heat flux, which, following Rennó et al. (1998), favors the formation of convective vortices (Newman et al. 2019).

Potential discrete dust clearing events could occur within seconds. Ordonez-Etxeberria et al. (2018) analyzed the behavior of the UV measurements during three pressure drops detected by REMS caused by dust devils, showing that the UV fluxes after the pressure drops were very similar to the previous ones. Due to the instrument accuracy, small variations in the amount of dust on the sensor have not been detected with sufficient certainty to date. However, the smoothed version of the DCF allows the analysis of the cumulated effect of those small dust clearing events, providing enhanced confidence in seasonal and interannual variations. The potential variations in the amount of dust accumulated on the REMS UV sensor associated to convective vortices will be assessed in a future study.

We have also performed the DCF calculations for the UVABC channel using Mastcam opacities at $440 \mathrm{~nm}$ (instead of those at $880 \mathrm{~nm}$ ) obtaining the same trend, with relative differences between $-2 \%$ and $+3 \%$. We selected the DCF obtained with Mastcam opacities at $880 \mathrm{~nm}$ due to their better temporal coverage. This is particularly important between sols 375 and 486, when the lack of opacity retrievals at 440 (Lemmon et al. 2019) would lead to a temporal gap in the DCF.

\subsection{Angular Response Correction}

We derive new angular response calibration functions using the same data as for the DCF, except for the fact that now we include measurements with solar zenith angles between $45^{\circ}$ and $55^{\circ}$ that meet the aforementioned requirements (solar position outside the field of view obstructed by the mast and masthead of the rover, masthead in the most typical position, Mastcam opacity retrievals available, and the rover being still).

We describe next the steps followed to derive the new angular response for each UVS channel. A simplified version of the methodology was presented in Vicente-Retortillo et al. (2017), where a new angular response for the UVE channel was obtained using measurements for the first 1413 sols of the MSL mission. Here, we extend the calculation of the angular responses to all channels using measurements during the first 2003 sols of the mission, and we improve the methodology by including the azimuthal dependence of the angular response.

First, we normalize the photodiodes output currents accounting for three factors: the temperature dependence of the spectral response, the Sun-Mars distance, and the atmospheric opacity. In addition, we divide the measurements by the DCF to correct them for the effects of dust accumulation on the sensor. Then, we create a table with grid cells separated by $1^{\circ}$ in solar zenith angle $(\theta)$ and $5^{\circ}$ in solar azimuth angle $(\phi)$. We store in each cell the mean of all the measurements performed with a solar position within $2^{\circ}$ of the position that defines the center of the cell, weighted by the inverse of that angular distance. Values for cells lacking measurements are obtained by solving the biharmonic equation using finite differences around those cells; then, values in the region that can be affected by shadows are obtained for each zenith angle by interpolating linearly using the values at the boundaries of the region. Since the flux at the surface after correcting for the effect of the atmosphere is controlled by $\cos (\theta)$, we divide the values of the table by the cosine of the solar zenith angle of each cell to obtain the angular response. Finally, in order to avoid spurious changes in the behavior of the corrected measurements, we smooth the values of the table using cubic smoothing splines. We note that this function represents the effective response of the sensor including all potential effects, accounting also for potential variations with solar position in the effect of deposited dust. 

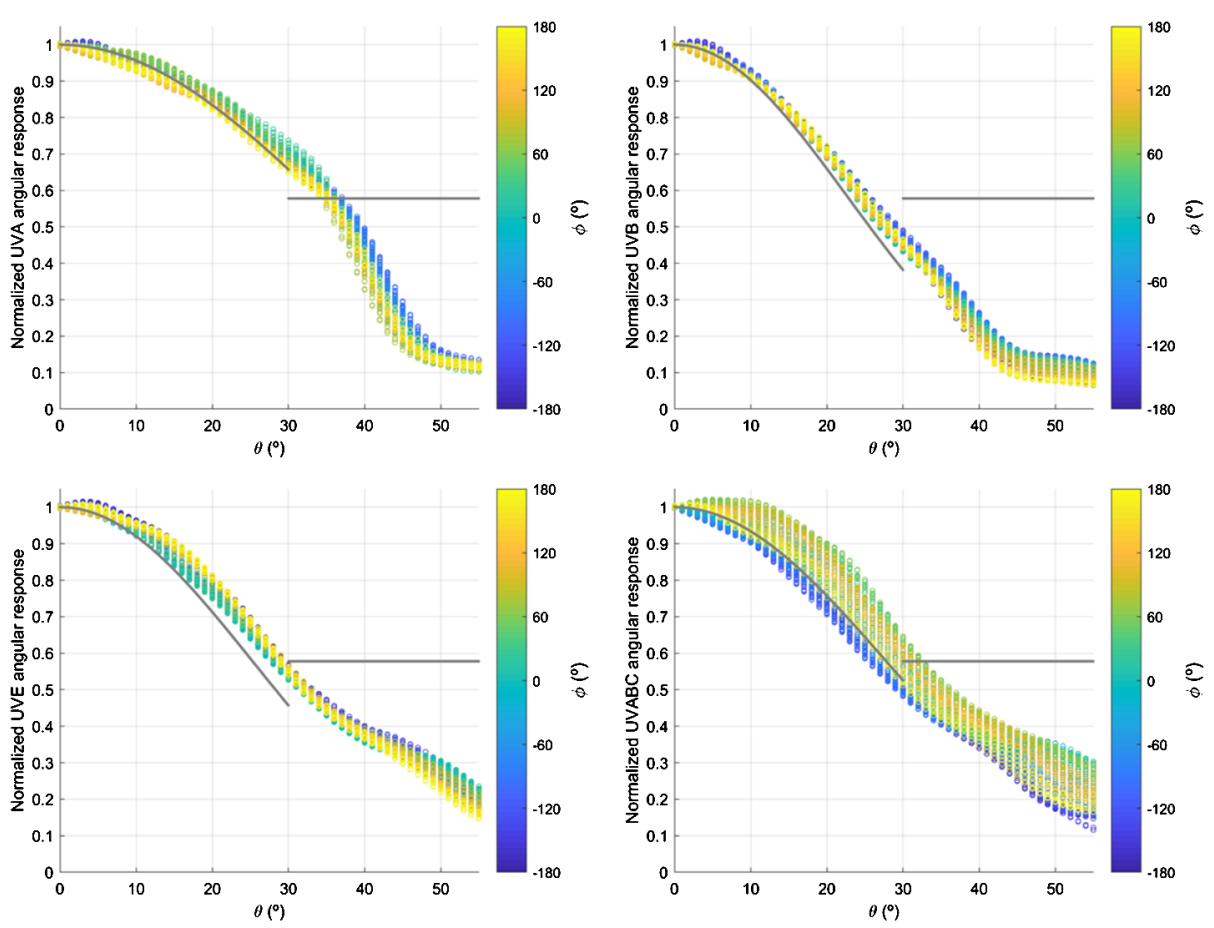

Fig. 6 Empirically derived angular response calibration functions as a function of zenith angle of UVA (top left), UVB (top right), UVE (bottom left) and UVABC (bottom right) channels. Lines are colored as a function of azimuth angle. For comparison, the angular response previously used is shown in gray

Figure 6 shows the new, empirically-derived angular responses for the UVA, UVB, UVE and UVABC channels as a function of zenith and azimuth angles. Channels UVC and UVD show similar behaviors, but with significantly larger azimuth dependence for solar zenith angles above $30^{\circ}$. For comparison, previous angular responses are shown in gray. The new angular responses are in good agreement with the previous functions for $\theta<30^{\circ}$, especially for $\theta<20^{\circ}$. Therefore, for measurements with $\theta<20^{\circ}$ (shown in blue in Fig. 4) the correction for the effects of dust deposition is generally the most significant. Exceptions to this rule occur when the DCF is close to 1, as well as for solar positions with larger differences between the previous and the new angular responses (such as for zenith angles close to $30^{\circ}$ or for angular responses with a high azimuth dependence).

In contrast, for $\theta>30^{\circ}$ there is a dramatic change in the behavior of previous and new angular responses. While the previous functions showed a discontinuity at $30^{\circ}$ and a constant value beyond, the new angular responses show a smooth behavior and their values depend on $\theta$ and $\phi$. The azimuth dependence is particularly important for large values of $\theta$ : for some channels and solar positions with low solar elevations, not considering the azimuthal dependence of the angular response of the sensor could lead to errors above $100 \%$.

Once we determine the effect of dust accumulated on the sensor and the new sensor's angular response, we convert the photodiode output currents to UV fluxes by dividing the former by the product of the responsivity of the sensor (obtained from Fig. 1, but including also its temperature dependence), the DCF (Fig. 5) and the angular response (Fig. 6). 
Fig. 7 UVABC fluxes on sol 1406 as currently stored in ENVRDR products (gray) and after the correction for the effects of dust deposition and sensors' angular response (red). Their relative difference is shown in black

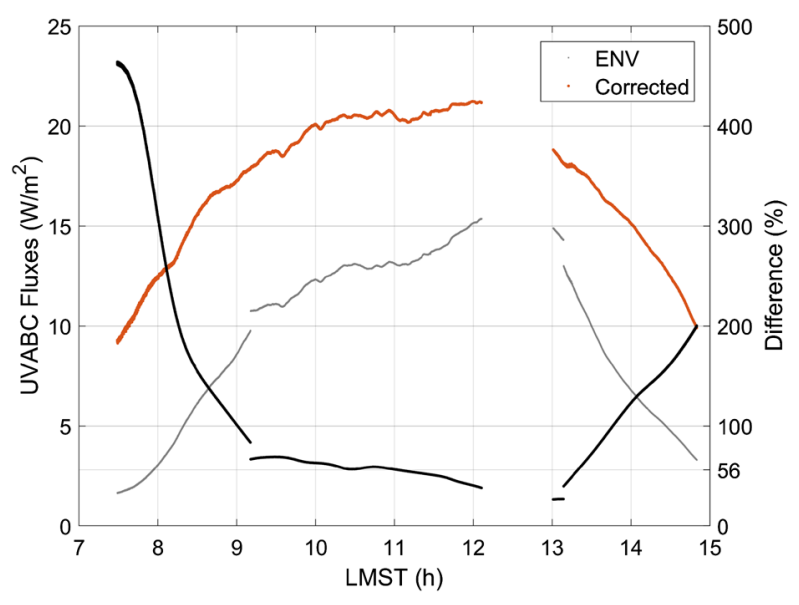

\section{Results}

\subsection{Corrected UV Fluxes}

As an example of the performance of the correction presented in this article, Fig. 7 shows in red UVABC fluxes corrected for the effects of dust deposition and sensor's angular response on sol 1406 of the mission (MY 33, $\mathrm{L}_{\mathrm{s}}=189^{\circ}$ ). For comparison, UV fluxes stored in the ENVRDR products are shown in gray. For $\theta<30^{\circ}$ (between the discontinuities in the gray curve shortly after 9 and 13 LMST), the corrected values are 56\% larger on average (black curve), indicating the strong effect of dust accumulation on the UVS. Moreover, within this period the relative difference between corrected and previous values changes considerably as a function of $\phi$, with the largest differences occurring in the morning (69\% at 9:30 LMST) and the lowest past noon ( $26 \%$ at 13 LMST). This is caused by the spatial response of the sensor: the solar azimuth angle changed from $-120^{\circ}$ (which corresponds to a low relative angular response, represented with blue circles in the fourth panel of Fig. 6) to $40^{\circ}$ (green circles) during this period. Beyond the discontinuities at $30^{\circ}$, relative differences are significantly larger and show a markedly increase with $\theta$, reaching values well above $100 \%$ for measurements before 9 LMST and after $~ 13: 45$ LMST.

To illustrate the magnitude of our correction over the full temporal coverage of the UVS, we show in Fig. 8 the relative differences between corrected and previous (ENVRDR) UV fluxes as a function of sol number and LMST for the UVABC channel. At around noon, relative differences are mostly controlled by the amount of dust accumulated on the sensor and thus show the lowest values at the beginning of the mission and around sols 1500-1600 (MY 33 at $\mathrm{L}_{\mathrm{s}} \sim 300^{\circ}$ ), when the DCF presented maximum values (see Fig. 5). In contrast, relative differences within a sol are controlled by the solar zenith angle and thus markedly increase with the distance to local noon throughout the mission.

We note that relative differences around noon can also be significant, depending mostly on the DCF and the maximum daily solar elevation. We find the best example around sol 850 (MY 32, $\mathrm{L}_{\mathrm{s}} \sim 260^{\circ}$ ), when large amounts of accumulated dust (see black curve in Fig. 5) and minimum daily $\theta$ above $38^{\circ}$ kept the differences above $100 \%$ throughout these sols. On average, relative differences are $\sim 35-40 \%$ for solar zenith angles below $30^{\circ}$ and above $100 \%$ for solar zenith angles above $37^{\circ}$. 


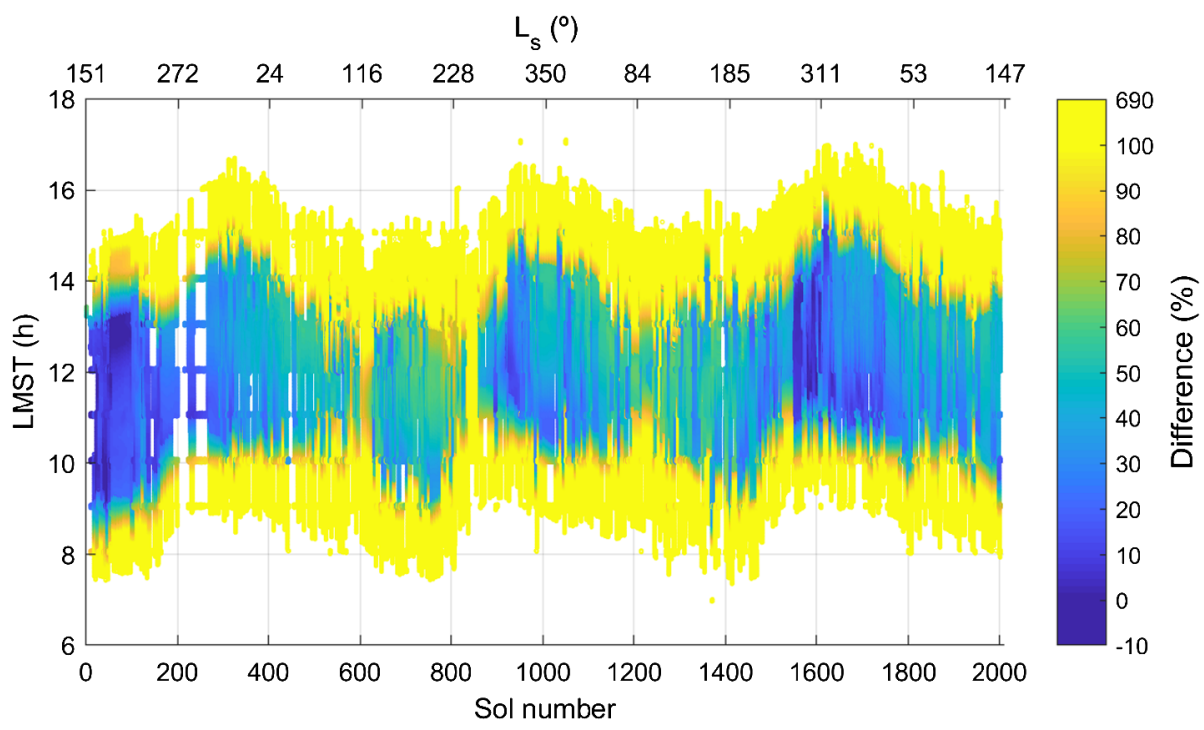

Fig. 8 Ratio between corrected and ENVRDR UV fluxes as a function of sol number and LMST for the UVABC channel
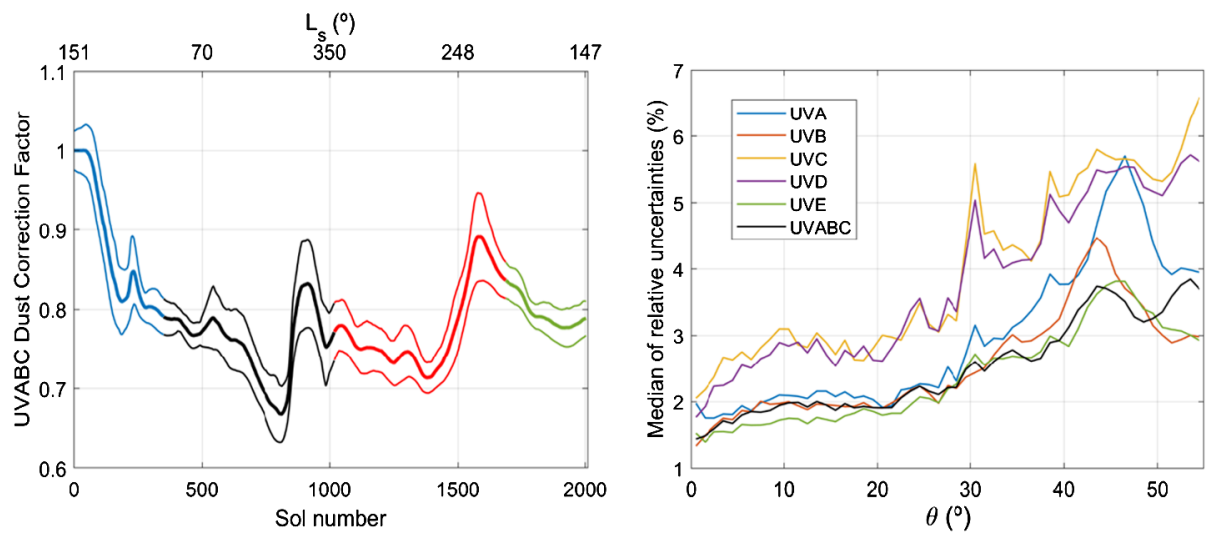

Fig. 9 (Left) Temporal evolution of the UVABC Dust Correction Factor (thick line) with its uncertainties (thin lines). Different colors represent different Mars years, as in Fig. 5. (Right) Median of the relative uncertainties as a function of solar zenith angle for the six REMS UV channels

\subsection{Determination of Uncertainties}

The main sources of uncertainties in the corrected fluxes arise from the DCF and new angular response functions. As described in Vicente-Retortillo et al. (2018), uncertainties in the DCF can be calculated from the mean and standard deviation of the differences between the DCF values before and after smoothing (see Sect. 3). Following a conservative approach, we obtain the uncertainties in DCF by adding the absolute value of the mean and the standard deviation of the differences.

The left panel of Fig. 9 shows the DCF and uncertainty of the UVABC channel. Relative errors are between $\sim 1.7 \%$ and $\sim 6.8 \%$, with a median of $4.0 \%$. The median is smaller for the 
remaining channels, except for the UVD. The UVE channel shows the lowest uncertainties, the median being 2.9\%; on the other extreme, the UVD channel shows a median of $6.1 \%$.

Additionally, we obtain the uncertainties associated to the new angular responses and to the performance of the sensors by comparing simultaneous corrected values of different channels. This method relies on the assumption that the behavior of the mean of several channels is expected to be more reliable than the behavior of each separate channel. To illustrate this, let us assume that five channels measure a small increase in the incoming radiation during a given period while the sixth channel measures a decrease. In absence of elements that could actually cause this behavior (such as the masthead starting to cast a shadow on that particular photodiode), the increase of radiation appears to be the most likely option and the measurements of the sixth channel appear to be less accurate. Following the methodology described below, we would assign a larger uncertainty to the sixth channel.

First, we discard measurements when the mast or masthead of the rover cast a partial shadow on at least one of the UVS channels. For each sol, we normalize the corrected values of the UVA, UVB, UVE and UVABC channels (UVC and UVD channels show a lower reliability, particularly for large solar zenith angles) and we add them. We then calculate the deciles and store the 11 measurements of the sol closest to those values. Then, we normalize the measurements of each channel with respect to the value at the first of the 11 selected measurements. We add the normalized values of the four selected channels and repeat the normalization for this additional variable (referred to as the mean). We then select the measurements within six minutes for each measurement and channel. We calculate the standard deviation of the differences between the selected measurements and those of the mean. We add it to the absolute value of the differences between the mean value of the selected measurements of that channel and the mean value of those of the mean. We divide the resulting value by the mean of the selected measurements of that channel, and repeat this process for each of the aforementioned 11 measurements. Finally, the 11 temporal series of relative uncertainties for each channel and sol are averaged, with relative uncertainties when measurements are flagged for being affected by partial shadows obtained by interpolation (when possible within each sol).

The right panel of Fig. 9 shows the median of the relative uncertainties associated to the spatial response and to the performance of the sensors for each channel as a function of the solar zenith angle. The median generally increases with solar zenith angle; however, while relative uncertainties peak at $\sim 55^{\circ}$ for channels UVC, UVD and UVABC, channels UVA, UVB and UVABC show the highest uncertainties between $40^{\circ}$ and $50^{\circ}$. This figure offers valuable information regarding the degree of confidence of the corrected values. For solar zenith angles below $40^{\circ}$, channels UVA, UVB, UVE and UVABC show similar values and behavior, with smaller uncertainties than UVC and UVD channels. For solar zenith angles between $40^{\circ}$ and $55^{\circ}, \mathrm{UVE}$ and UVABC channels show the lowest uncertainty.

Finally, we calculate the total absolute uncertainty $\left(\mathrm{W} / \mathrm{m}^{2}\right)$ in the corrected UV fluxes by taking the root sum of the squares of the uncertainties arising from the DCF and new angular responses, and then multiplying by the corrected UV flux. As an example, Fig. 10 shows the diurnal evolution of corrected UVABC fluxes (red curve) on sol 1406, along with the associated uncertainties (gray curves).

\section{Robustness of Generated UV Fluxes}

In order to assess the robustness of the corrected fluxes, we have performed tests to validate the three functions that are involved in the conversion from photodiode output currents 
Fig. 10 Corrected UVABC fluxes on sol 1406 (red curve) and the associated uncertainty (gray curves)

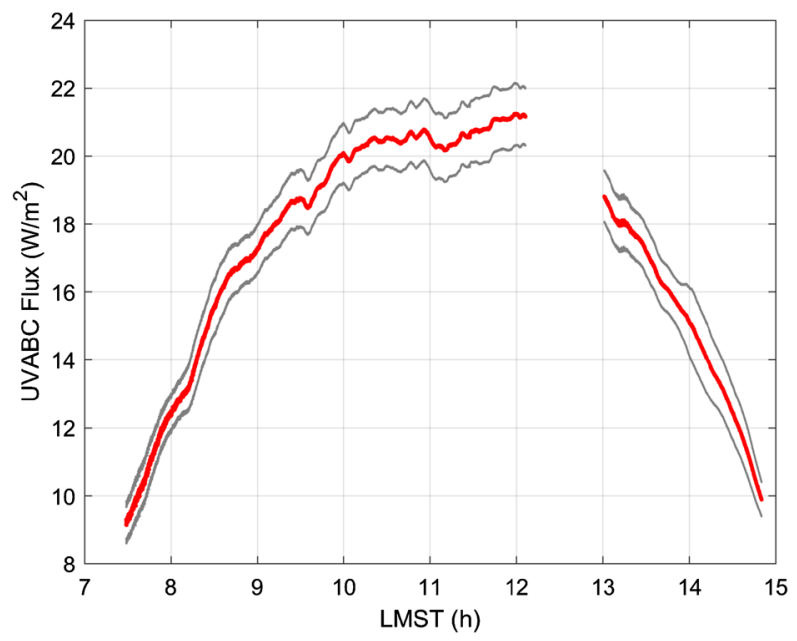

Table 1 Median of observed and expected ratios to the UVABC channel, and ratio between both of them

\begin{tabular}{llllll}
\hline & UVA & UVB & UVC & UVD & UVE \\
\hline Observed & 0.675 & 0.314 & 0.077 & 0.158 & 0.498 \\
Expected & 0.708 & 0.327 & 0.105 & 0.147 & 0.536 \\
Obs/Exp & 0.954 & 0.961 & 0.737 & 1.081 & 0.930 \\
\hline
\end{tabular}

to corrected UV fluxes: the spectral response, the dust correction factor and the angular response.

In order to validate the spectral responses, we compare the observed ratios of corrected fluxes between each channel and the UVABC channel to what is expected when accounting for the DCF, the angular responses and Mastcam opacities. The expected measurements are calculated as follows:

$$
M_{\text {exp }}=D C F \cdot A R \cdot \int E \cdot S R \cdot d \lambda
$$

In this equation, $E$ is the spectral UV irradiance, simulated with the radiative transfer model COMIMART (Vicente-Retortillo et al. 2015), fed with Mastcam opacities (Lemmon et al. 2019), and $A R$ and $S R$ are the angular and spectral responses of the sensor, respectively. Then, for each channel, we have calculated hourly values of the ratio between its expected measurements and those of the UVABC channel.

Table 1 shows the median of the hourly values of the observed and expected ratios between the corrected values of each channel and those of the UVABC channel, as well as the ratio between both of them. The observed ratios between channels are in good agreement with the expected values, with differences comparable to the uncertainties of the measurements. The exception is the UVC channel, where observations are significantly lower than the expected values; in any case, it is still possible to analyze the ratio between the UVC and UVABC channels by dividing it by 0.737 and using the spectral responses of Fig. 1.

In order to assess the robustness of the dust corrections, we calculate the correlations among the DCF for each pair of channels. Figure 11 shows these correlations, indicating a good overall agreement among the channels. Correlation coefficients, indicated in the cor- 


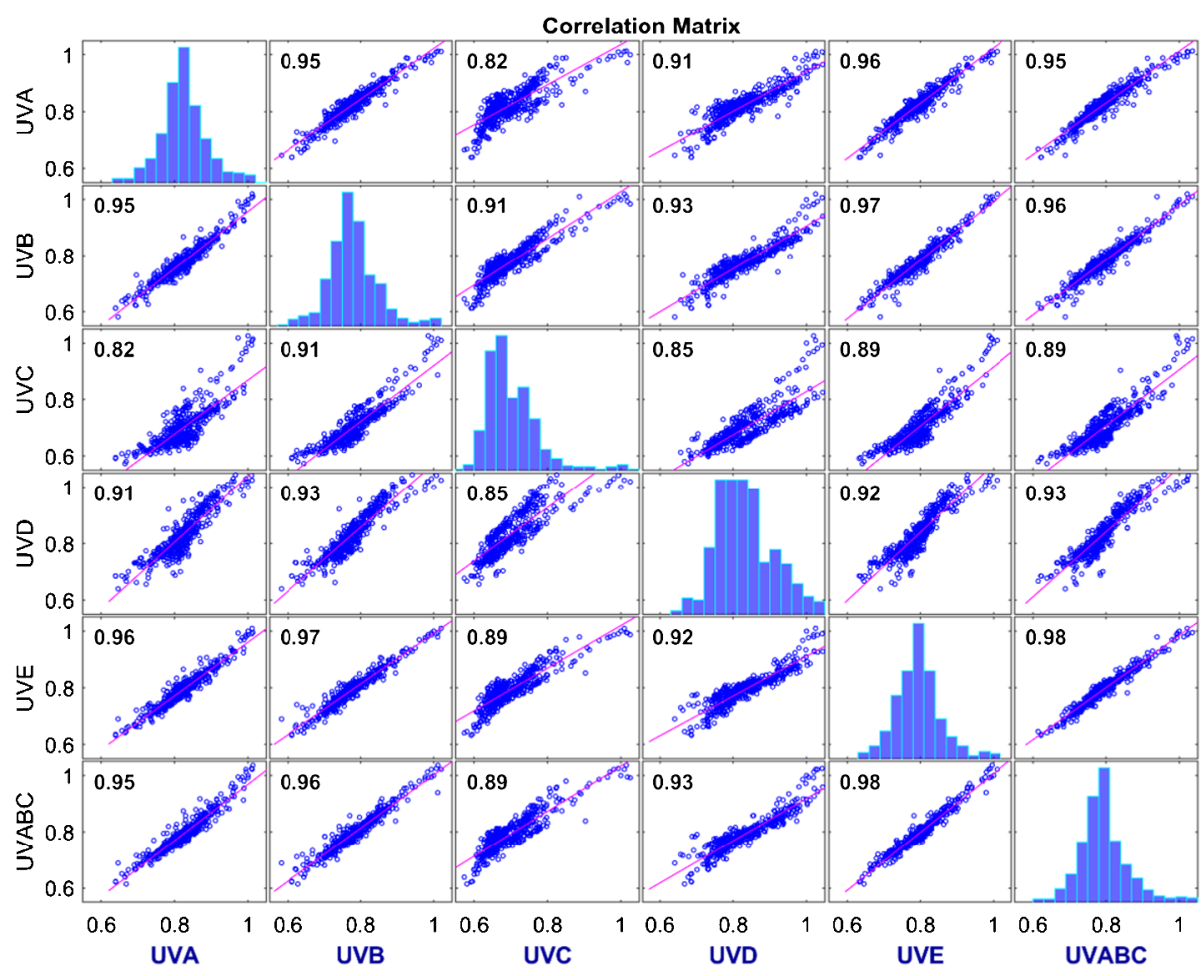

Fig. 11 Correlations among DCFs for each pair of channels. Correlation coefficients are shown in each correlation plot. The diagonal of the matrix of plots shows the histograms for each channel

relation plots, are particularly high for the four channels with the highest confidence (UVA, UVB, UVE and UVABC), while they are lower for UVC and UVD.

In order to assess the robustness of the angular response corrections, we perform comparisons among the six channels as a function of $\theta$ and $\phi$. As an example, the left panel of Fig. 12 shows the ratio of UVE to UVA fluxes stored in the ENVRDR products as a function of solar zenith and azimuth angles. This ratio shows a sharp decrease at $\theta=30^{\circ}$ that is caused by the discontinuity in the corresponding angular response calibration function, which affects both channels differently (Fig. 6, left panels). This ratio becomes significantly smaller for $30^{\circ}<\theta<40^{\circ}$, while this behavior reverses for $45^{\circ}<\theta<55^{\circ}$, where UVE values are higher than those of the UVA channel.

The right panel of Fig. 12 shows the ratio of the same channels, but now using the values corrected for the effects of dust deposition and inaccuracies in the angular response. The large variations as a function of $\theta$ that are present in the ENVRDR products are virtually absent in the corrected data. Moreover, the more modest variations in the ratio as a function of $\phi$ introduced by the angular response of the photodiodes have been virtually removed in the corrected data. The values that differ the most from the typical ratios are found in the region at $-100^{\circ}<\phi<10^{\circ}$ which is close to the edges of the region of the field of view that is blocked by the masthead and the mast of the rover, and are an indicator of actual differences in the relative position of the rover elements with respect to the photodiodes. 

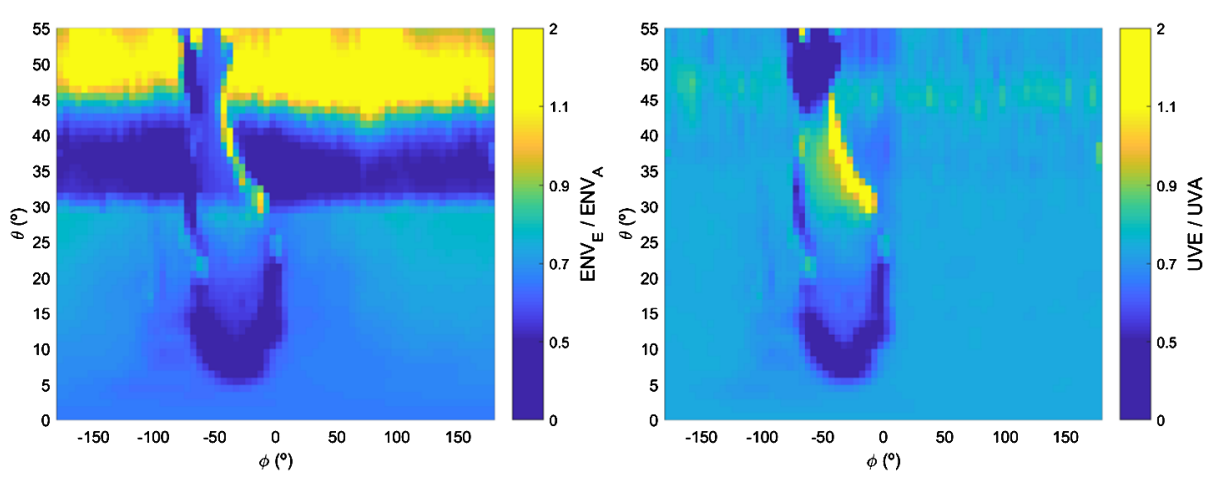

Fig. 12 (Left) Ratio of UVA and UVE ENVRDR products as a function of solar zenith $(\theta)$ and azimuth $(\phi)$ angles. (Right) Ratio of UVA and UVE fluxes after applying the dust correction factor and the new empirical angular response functions. The region with extreme values at $-100^{\circ}<\phi<10^{\circ}$ is affected by mast shadows

\section{Summary and Conclusions}

Measurements by the REMS/UVS onboard the MSL Curiosity rover constitute the first in situ dataset of UV radiation flux at the surface of Mars. The UVS is comprised of six photodiodes to measure in six different bands (Fig. 1). At the time of this writing, the UVS has completed more than 4.1 MYs of measurements, providing coverage from diurnal to interannual scales.

Due to its position on the Curiosity deck, the UVS has been exposed to dust deposition (Fig. 2). This dust causes underestimation in measured UV fluxes. In addition, inaccuracies in the original angular response calibration functions of the UVS have led to discrepancies between measured and physically-consistent UV fluxes when the solar zenith angle relative to the rover frame is between $20^{\circ}$ and $55^{\circ}$ (Fig. 3). This is important because measurements acquired when $20^{\circ}<\theta<55^{\circ}$ represent $\sim 45 \%$ of the whole set of UVS data with $\theta<90^{\circ}$ (Fig. 4). Moreover, the original angular responses did not consider dependence on the solar azimuth angle, which in some cases can lead to significant inaccuracies in measured UV flux.

In this article we present a methodology to correct MSL/REMS UV fluxes when $\theta<55^{\circ}$ for both effects (Figs. 5 and 6), and show results of the corrected data set for the first 2003 sols ( $\sim 3 \mathrm{MYs})$ of the MSL mission, from $\mathrm{L}_{\mathrm{s}} \sim 151^{\circ}$ in MY 31 to $\mathrm{L}_{\mathrm{s}} \sim 149^{\circ}$ in MY 34 . Close to noon, when $\theta$ values are typically $<30^{\circ}$, relative differences between corrected and original UV fluxes are $\sim 35-40 \%$ on average (Fig. 8). These differences are primarily caused by the amount of dust accumulated on the sensor, with a weaker but still significant contribution from changes in the spatial response as a function of the solar zenith and azimuthal angles. Outside hours close to noon, when $\theta$ is typically $>30^{\circ}$, relative differences are above $>100 \%$ (Fig. 8). In this case, they are mostly caused by differences between the new and original angular responses as a function of $\theta$ and $\phi$, with lesser contribution from changes in accumulated dust.

The whole set of corrected UV fluxes presented in this article, along with the associated uncertainty (Fig. 9) and the information on the geometry of the rover and the Sun, are available in the NASA PDS for each of the six UVS channels (https://atmos.nmsu.edu/PDS/data/ mslrem_1001/DATA_UV_CORRECTED/). This new dataset does not replace UV fluxes contained in the ENVRDR and MODRDR products (highest-order products available in the PDS; Sect. 2), but is posted in a separate folder (UVRDR products). We plan to upload 
corrected UV fluxes beyond sol 2003 in future releases, including those during 2018/MY34 global dust storm.

UV fluxes generated in this study are important to study the effect of UV radiation on the variability of major $\left(\mathrm{CO}_{2}, \mathrm{~N}_{2}, \mathrm{Ar}, \mathrm{O}_{2}\right.$ and $\left.\mathrm{CO}\right)$ and minor $\left(\mathrm{CH}_{4}\right)$ atmospheric species, to recreate accurate UV doses for biological laboratory experiments, to perform combined analyses of satellite and ground-based measurements, and to allow comparisons of the UV radiation environment at different landing locations.

Acknowledgements This research was supported by Jet Propulsion Laboratory grant number 1449038 and LPI/USRA Subaward No. Subk00011877. The fluxes correction here presented would not have been possible without the previous effort of and support from the MSL team, in particular REMS team members. We would like to thank Michael Smith and Jeffrey R. Johnson for their excellent suggestions to improve the manuscript. Corrected UV fluxes shown in this manuscript are available in the Atmospheres Node of the Planetary Data System. Lower-order UV data (ADR and TELRDR products) used to obtain corrected UV fluxes are also available in the NASA PDS.

Publisher's Note Springer Nature remains neutral with regard to jurisdictional claims in published maps and institutional affiliations.

Open Access This article is licensed under a Creative Commons Attribution 4.0 International License, which permits use, sharing, adaptation, distribution and reproduction in any medium or format, as long as you give appropriate credit to the original author(s) and the source, provide a link to the Creative Commons licence, and indicate if changes were made. The images or other third party material in this article are included in the article's Creative Commons licence, unless indicated otherwise in a credit line to the material. If material is not included in the article's Creative Commons licence and your intended use is not permitted by statutory regulation or exceeds the permitted use, you will need to obtain permission directly from the copyright holder. To view a copy of this licence, visit http://creativecommons.org/licenses/by/4.0/.

\section{References}

I. Arruego et al., in Sun Irradiance and Dust Sensor Investigations on Board the ExoMars 2018 Lander. International Planetary Probe Workshop (IPPW-13), Laurel, MD, USA (2016)

J. Gómez-Elvira, C. Armiens, L. Castañer, M. Domínguez, M. Genzer, F. Gómez et al., REMS: The environmental sensor suite for the Mars Science Laboratory rover. Space Sci. Rev. 170(1-4), 583-640 (2012)

J. Gómez-Elvira, C. Armiens, I. Carrasco, M. Genzer, F. Gómez, R. Haberle et al., Curiosity's rover environmental monitoring station: Overview of the first 100 sols. J. Geophys. Res., Planets 119, 1680-1688 (2014)

S.D. Guzewich, M. Lemmon, C.L. Smith, G. Martínez, Á. Vicente-Retortillo, C.E. Newman et al., Mars Science Laboratory Observations of the 2018/Mars Year 34 global dust storm. Geophys. Res. Lett. 46(1), 71-79 (2019)

J.R. Johnson, J.F. Bell III, S. Bender, D. Blaney, E. Cloutis, L. DeFlores, B. Ehlmann, O. Gasnault, B. Gondet, K. Kinch, M. Lemmon et al., ChemCam passive reflectance spectroscopy of surface materials at the Curiosity landing site, Mars. Icarus 249, 74-92 (2015)

M.T. Lemmon, S.D. Guzewich, T. McConnochie, A. de Vicente-Retortillo, G.M. Martinez, M.D. Smith, J.F. Bell III, D. Wellington, S. Jacob, Large dust aerosol sizes seen during the 2018 Martian global dust event by the Curiosity rover. Geophys. Res. Lett. 46, 9448-9456 (2019)

M.B. Madsen et al., Magnetic Properties Experiments on the Mars Exploration Rover mission. J. Geophys. Res., Planets 108(E12), 8069 (2003). https://doi.org/10.1029/2002JE002029

T.H. McConnochie et al., Retrieval of water vapor column abundance and aerosol properties from ChemCam passive sky spectroscopy. Icarus 307, 294-326 (2018)

C.E. Newman, H. Kahanpää, M.I. Richardson, G.M. Martinez, A. Vicente-Retortillo, M.T. Lemmon, MarsWRF convective vortex and dust devil predictions for gale crater over 3 Mars years and comparison with MSL-REMS observations. J. Geophys. Res., Planets 124, 3442-3468 (2019)

I. Ordonez-Etxeberria, R. Hueso, A. Sánchez-Lavega, A systematic search of sudden pressure drops on Gale crater during two Martian years derived from MSL/REMS data. Icarus 299, 308-330 (2018)

N.O. Rennó, M.L. Burkett, M.P. Larkin, A simple thermodynamical theory for dust devils. J. Atmos. Sci. 55, 3244-3252 (1998) 
J.A. Rodriguez-Manfredi et al., MEDA: An environmental and meteorological package for Mars 2020, in 45th Lunar and Planetary Science Conference (2014), p. 2837

M.D. Smith, M.P. Zorzano, M. Lemmon, J. Martín-Torres, T. Mendaza de Cal, Aerosol optical depth as observed by the Mars Science Laboratory REMS UV photodiodes. Icarus 280, 234-248 (2016)

M.G. Trainer et al., Seasonal variations in atmospheric composition as measured in Gale Crater, Mars. J. Geophys. Res., Planets 124, 3000-3024 (2019)

Á. Vicente-Retortillo, F. Valero, L. Vázquez, G.M. Martínez, A model to calculate solar radiation fluxes on the Martian surface. J. Space Weather Space Clim. 5, A33 (2015)

A. Vicente-Retortillo, G.M. Martínez, N.O. Renno, M.T. Lemmon, M. de la Torre-Juárez, Determination of dust aerosol particle size at Gale Crater using REMS/UVS and Mastcam measurements. Geophys. Res. Lett. 44, 3502-3508 (2017)

Á. Vicente-Retortillo, G.M. Martínez, N. Renno, C.E. Newman, I. Ordonez-Etxeberria, M.T. Lemmon, M.I. Richardson, R. Hueso, A. Sánchez-Lavega, Seasonal deposition and lifting of dust on Mars as observed by the Curiosity rover. Sci. Rep. 8(1), 17576 (2018)

C.R. Webster et al., Background levels of methane in Mars' atmosphere show strong seasonal variations. Science 360(6393), 1093-1096 (2018)

M.J. Wolff, R.T. Clancy, J.D. Goguen, M.C. Malin, B.A. Cantor, Ultraviolet dust aerosol properties as observed by MARCI. Icarus 208(1), 143-155 (2010) 